\title{
Genome-wide analysis of epigenetic dynamics across human developmental stages and tissues
}

\author{
Xia Zhang ${ }^{1 \dagger}$, Yanglan Gan ${ }^{2 *}$, Guobing Zou ${ }^{1}$, Jihong Guan ${ }^{3}$ and Shuigeng Zhou ${ }^{4}$ \\ From The 17th Asia Pacific Bioinformatics Conference (APBC 2019) \\ Wuhan, China. 14-16 January 2019
}

\begin{abstract}
Background: Epigenome is highly dynamic during the early stages of embryonic development. Epigenetic modifications provide the necessary regulation for lineage specification and enable the maintenance of cellular identity. Given the rapid accumulation of genome-wide epigenomic modification maps across cellular differentiation process, there is an urgent need to characterize epigenetic dynamics and reveal their impacts on differential gene regulation.

Methods: We proposed DiffEM, a computational method for differential analysis of epigenetic modifications and identified highly dynamic modification sites along cellular differentiation process. We applied this approach to investigating 6 epigenetic marks of 20 kinds of human early developmental stages and tissues, including hESCs, 4 hESC-derived lineages and 15 human primary tissues.

Results: We identified highly dynamic modification sites where different cell types exhibit distinctive modification patterns, and found that these highly dynamic sites enriched in the genes related to cellular development and differentiation. Further, to evaluate the effectiveness of our method, we correlated the dynamics scores of epigenetic modifications with the variance of gene expression, and compared the results of our method with those of the existing algorithms. The comparison results demonstrate the power of our method in evaluating the epigenetic dynamics and identifying highly dynamic regions along cell differentiation process.
\end{abstract}

Keywords: Epigenetic modification, Differential analysis, Hamming distance

\section{Background}

Lineage specification and maintenance of cellular identity are complex biological processes [1]. It is now widely accepted that cell phenotypes are significantly regulated by epigenetic states and that chromatin changes during differentiation contribute to the determination of cell fate [2]. Recent evidence further shows that coordinated epigenetic changes influence the maintenance of such cellular memory [3, 4]. DNA methylation and certain epigenetic modifications are essential for chromatin structures and

*Correspondence: ylgan@dhu.edu.cn

${ }^{\dagger}$ Xia Zhang and Yanglan Gan contribute equally to the paper.

${ }^{2}$ School of Computer Science and Technology, Donghua University, Shanghai,

China

Full list of author information is available at the end of the article gene expression in proper execution of developmental programs $[5,6]$. Therefore, a fundamental question in the field is to exactly answer where and how the epigenetic changes regulate phenotypic changes.

To fully understand the dynamics and regulatory roles of epigenetic modifications, advanced sequencing technologies have generated genome-wide epigenetic maps of diverse developmental stages, lineages and tissues [7, 8]. In previous studies, researchers have differentiated human embryonic stem cells (hESCs) into mesendoderm, neural progenitor cells, trophoblast-like cells, and mesenchymal stem cells and systematically sequenced the transcriptome and epigenetic modifications of these lineages $[9,10]$. The first three hESC derivatives reflects critical developmental linages in the embryo [11]. Mesenchymal stem 
cells have the ability of further multi-lineage differentiation to bone, cartilage, adipose, muscle, and connective tissues [12]. Mouse embryonic stem cells were also differentiated into a variety of precursor cell types [13]. The expanding body of epigenomic data permits researchers to study the dynamics of epigenetic marks. This is a key step to reveal regulatory roles of epigenetic modifications, and to understand how global features of epigenetic modifications impact cellular phenotypes across different developmental stages, lineages and tissues.

Most previous works focused on comparing the epigenetic modification profiles between two biological conditions, and further identifying regions that show differential patterns, such as ChIPDiff [14], diffReps [15], dPCA [16], HistoneHMM [17], csaw [18] and HMCan-diff [19]. While some other methods such as dMCA [20] and Yang's method [21], were designed to detect cell-type-specific differential regions. Moreover, there are also some methods that were designed for identifying differential methylated region, such as QDMR [22] and MethylAction [23], whereas QDMR can also be applied to histone modification data analysis. Although several algorithms have been developed to analyze the epigenetic difference between two different conditions, little work devoting to differential analysis of epigenetic modifications among multiple cell types and across different developmental stages.

Here, we presented DiffEM, a computational method to quantify the dynamics of epigenetic marks and identified highly dynamic modification sites (HDMSs) across different human embryonic developmental stages. We applied this method to a public datasets with 6 intensely studied epigenetic marks of 20 different developmental stages and tissues. We identified HDMSs where different cell types exhibit distinctive epigenetic modification patterns, and found that these highly dynamic sites are enriched in genes related to cellular development and differentiation. We further correlated the dynamics scores of these epigenetic marks with those of gene expression levels. The results indicate that the changes of gene expression are closely related to the modification patterns of H3K4me1 and H3K27me3 in promoter regions during cell differentiation process. We compared DiffEM with the existing algorithms for identifying HDMSs. The comparison results show that DiffEM perform better in evaluating the epigenetic dynamics and identifying highly dynamic modification sites. This method is promising for broad applications in evaluating epigenetic dynamics in other complex biological processes.

\section{Materials and methods Datasets}

To analyze the dynamic epigenetic changes during cellular differentiation and lineage specification, we obtained a large panel of epigenetic maps of human embryonic stem cells (hESCs) and the key derivatives, including trophoblast-like cells (TBL), mesendoderm (ME), neural progenitor cells (NPCs), and mesenchymal stem cells (MSCs). The iHMS [24] database has integrated massive genome-wide epigenetic modification maps and RNA expression data spanning different developmental stages and tissues. From iHMS, we downloaded 6 epigenetic modification maps (H3K4me1, H3K4me3, H3K9me3, H3K27ac, H3K27me3 and H3K36me3) of 20 different human developmental stages and tissues, including hESCs, the hESC-derived precursor cell types ( TBL, ME, NPCs and MSCs), and 15 human primary tissues (adipose, adrenal gland, adult liver, aorta, esophagus, gastric, left ventricle, lung, ovary, pancreas, psoas muscle, right ventricle, right atrium, sigmoid colon, spleen, thymus, small intestine, breast, brain and bladder). Meanwhile, the RNA expression data and reference gene annotations were also downloaded from iHMS.

\section{Overview of the DiffEM model}

To characterize epigenetic dynamics across different development stages, we developed DiffEM, a new method to estimate the dynamics of epigenetic modifications based on hamming distance and identify highly dynamic modification sites. Unlike the previous work [20], we aimed to detect highly dynamic regions of epigenetic modification during cell differentiation process. To evaluate the dynamics across different differential stages and the primary tissues respectively, these 20 cell types were further categorized into three groups, hESC-derived precursor cell types, primary tissues and the whole group. We introduce the following steps to identify HDMSs, which are also shown in Fig. 1.

Data binarization. The raw ChIP-seq data were preprocessed in iHMS database [24]. The whole-genome was first segmented into 200bp bins. For each bin, neighboring read counts were summarized into an integer, indicating the extent of epigenetic modification in this region [25]. To reduce the effect of noise, we transformed these integers into binary values. First, we calculated the binarization threshold, by dividing the total read counts of all bins by the number of bins. If the read count of a bin is higher than the threshold, its binary value is set as 1 , otherwise 0 , After binarization, we noticed that some bins have no signals in all cell types, which may consist of sequences of low mappability. The consecutive regions with length more than $5 \mathrm{~kb}$ were removed from the genome. Finally, for the 6 investigated epigenetic marks, we obtained 6 binary matrices $B_{K}$ of size $T$ (the number of cell types) by $N$ (the number of $200 \mathrm{bp}$ bins on the whole genome).

Calculation of the dynamics scores for each epigenetic mark. After data binarization, we calculated the dynamics scores for each epigenetic mark among multiple cell types. In particular, we used the hamming distance to measure 


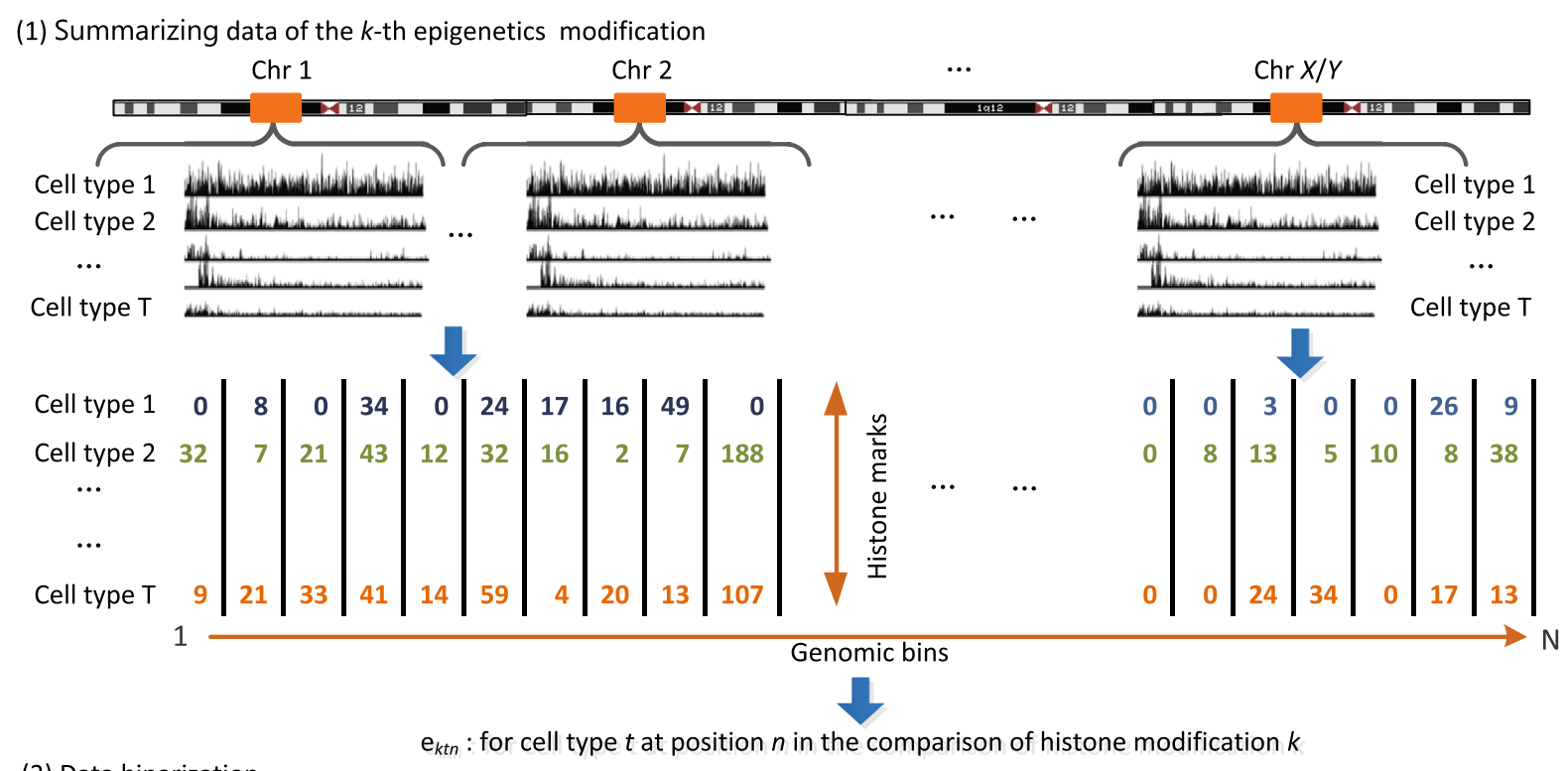

(2) Data binarization

$$
\text { Threshold }=\frac{\text { total read count }}{\text { the number of } 200 \text { bp bins }}
$$

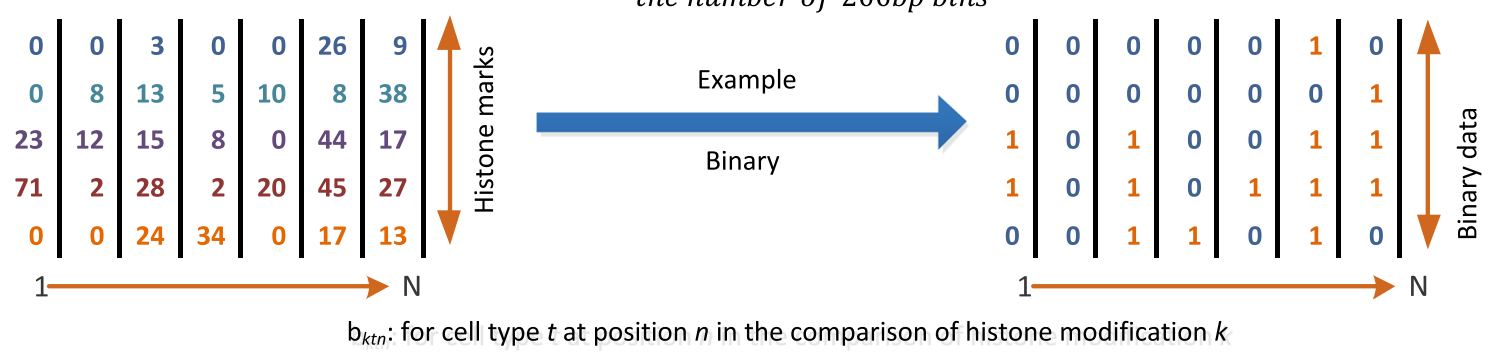

(3) Calculating the dynamics score of each epigenetic mark

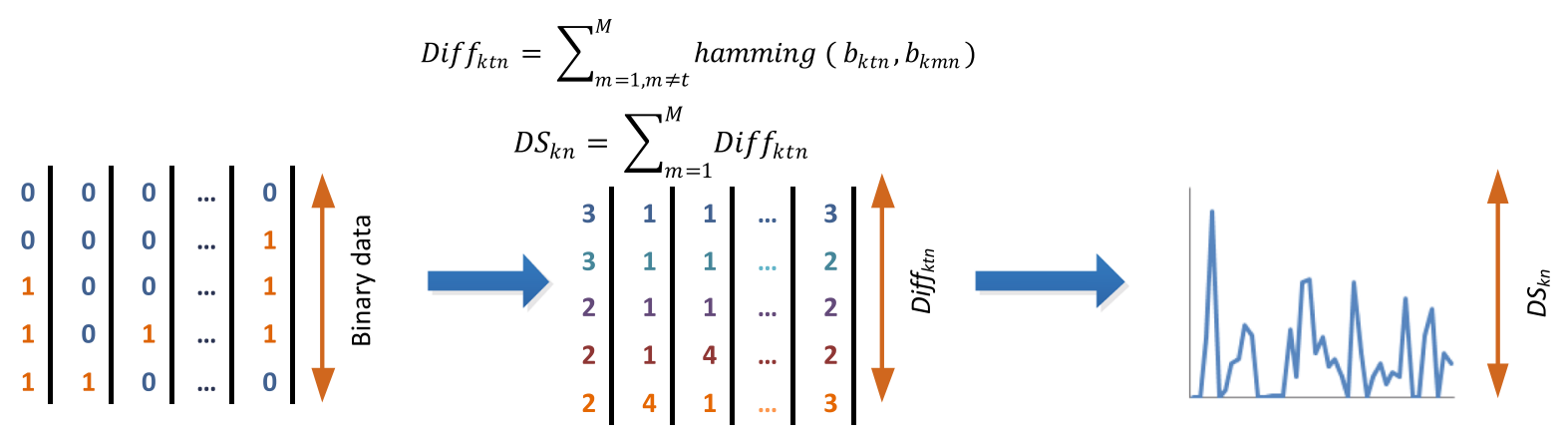

$D S_{\mathrm{kn}}$ : the dynamics score of epigenetic modification $k$ at position $n$

(4) Identifying the highly dynamic modification sites

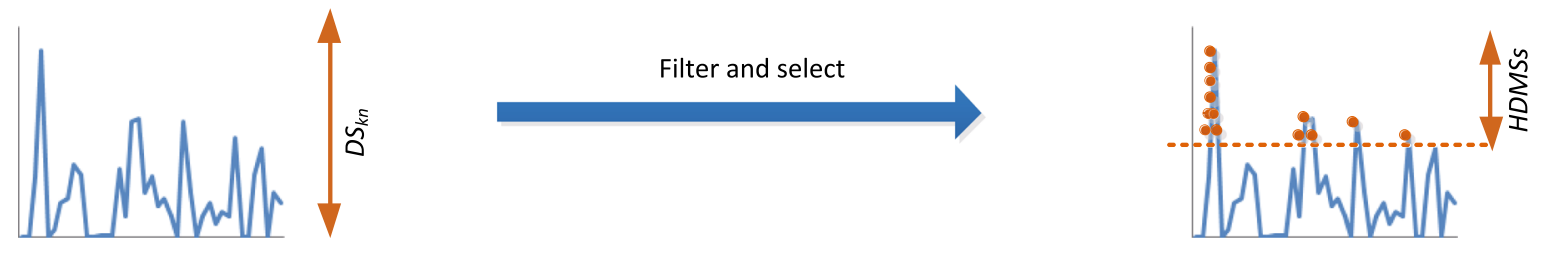

Fig. 1 The flowchart of the DiffEM approach 
the dynamics of each epigenetic modification. Here, we respectively calculated the dynamics scores of the 6 investigated epigenetic modifications in three cell type groups. As described above, given $M$ cell types and $N$ bins, we denoted $b_{k t n}$ as the binary profiles of epigenetic modification $k$ for cell type $t$ at position $n$. Then the difference between cell type $t$ and others are calculated as:

$$
\operatorname{Diff}_{k t n}=\sum_{m=1, m \neq t}^{M} \operatorname{hamming}\left(b_{k t n}, b_{k m n}\right)
$$

Further, the dynamics score of epigenetic modification $k$ at position $n$ was summed as:

$$
D S_{k n}=\sum_{t=1}^{M} \text { Diff }_{k t n}
$$

Identification of the highly dynamic modification sites. For each epigenetic mark, we have obtained the dynamics scores along the genome in each cell type group. The higher the dynamics score is, the greater the difference across these cell types exhibits. The sites with zero score were filtered first. Based on the calculated dynamics scores, we selected those bins whose dynamics scores are significantly higher than the genome background ( $p$ $<0.05$ ) and merged the adjacent bins into longer regions. These regions are referred to as highly dynamic modification sites (HDMSs).

\section{Functional analysis of the highly dynamic modification sites}

To investigate the potential functions of these identified HDMSs, we mapped them to RefSeq genes and some functional regions. According to their relative positions, we related the HDMSs to various genes when the centers of HDMSs are located in gene regions. The number of genes related to HDMSs was counted. Furthermore, we mapped the bins with the highest score to genomic features like promoter, coding region and exon. If a HDMS is not related to any gene, it is labeled as an intergenic sites. For further analysis of the functional relevance of HDMSs, we performed gene ontology (GO) enrichment analysis and pathway enrichment analysis for genes enriched with HDMSs via DAVID bioinformatics resources. The significant enrichment lists are obtained with $p<0.05$.

\section{Comparisons among different epigenetic modifications}

Epigenetic modifications play a critical role in cell differentiation process. Different epigenetic modifications may collaborate with each other to execute specific functions. We investigated the relations among different types of epigenetic marks. Based on the identified HDMSs of each epigenetic mark, the common HDMSs between different epigenetic modifications were obtained in the whole genome. Further, we estimated the correlations between the dynamics scores of these epigenetic modifications.

\section{Correlation analysis between the dynamics of epigenetic mark and gene expression}

First, we evaluated the dynamic scores of gene expression along the genome in each cell type group, which was calculated as the variance divided by the mean of gene expression. Then, we evaluated the correlation coefficients between the dynamic scores of epigenetic modifications and gene expression levels. For those identified HDMSs, a higher correlation coefficient indicates that gene expression is more easily regulated by the specific epigenetic modification.

\section{Comparison among DiffEM, QDMR and IOD}

As there exists no gold standard to benchmark highly dynamic modification sites, we adopted an indirect validation strategy. As previous studies [26], the validation was based on the correlations between the dynamics of epigenetic modifications and gene expression levels. To evaluate the performance in identifying HDMSs, we compared DiffEM with existing methods, QDMR and IOD. Unlike the methods restricted to the differential analysis between two cell types, the above three methods are capable of analyzing three or more cell types. QDMR was proposed for genome-wide differential analysis of epigenetic states based on Shannon entropy [22]. IOD was developed to detect differential regions across multiple cell types [27]. We first normalized the epigenetic data, and used QDMR and IOD to detect highly dynamic modification sites. These methods were compared by the correlations between the dynamics of epigenetic modifications and expression levels of the HDMSs.

\section{Results}

To investigate the dynamics of epigenetic modifications during cell differentiation process, we proposed a computational method, DiffEM, to quantify the dynamics score of various epigenetic marks and identify highly dynamic modification sites (HDMSs). We focused on human differentiation-related cell types, consisting of human embryonic stem cell, 4 hESC-derived precursor cell types, and 15 primary tissues. In each cell type, we collected 6 genome-wide epigenetic maps and gene expression datasets. DiffEM was applied to identify HDMSs along cell differentiation process. To evaluate the performance of our proposed method, in this section we analyzed the identified HDMSs to discover their potential biological roles during cell differentiation and development. Furthermore, we compared DiffEM with two previous methods, QDMR and IOD.

\section{Genome-wide characterization of epigenetic modification dynamics}

To better explore the dynamic epigenetic changes across different cell differentiation stages, these 20 cell types 
were further grouped into three groups, hESCs and hESCderived precursor cell types, primary tissues and the whole group. For each group and each epigenetic modification mark, we quantified the dynamics score for each bin based on hamming distance, and then ranked these bins according to their dynamics scores. We selected those bins whose dynamics scores were significantly higher than the genome background $(p<0.05)$.

After merging the neighboring bins, we obtained the HDMSs for each epigenetic modification in each group. For different epigenetic marks, we found that there exist big overlaps between the HDMSs of different epigenetic modifications. This is consistent with previous finding that the epigenetic modifications collaborated with each other to consummate certain regulatory function. As shown in Fig. 2, we respectively calculated the percentage of overlapping HDMSs among 6 epigenetic modifications in these three groups. On the whole, the overlapping sites make up $20 \% \approx 0 \%$ of total HDMSs in different groups. In the hESCs and hESC-derived precursor group, the HDMSs of different epigenetic marks overlap more than those of the other two groups. For example, the overlap rates of $\mathrm{H} 3 \mathrm{~K} 4 \mathrm{me} 1$ with other five epigenetic marks range from $40 \%$ to $50 \%$ in hESC-derived group, while those overlap rates in the other two groups are not greater than $25 \%$. Specifically, H3K4me3 is highly overlapped with H3K9me3 and H3K27ac. These observations demonstrate that epigenetic modifications collaborate closely to regulate the cell differentiation process [4].

As distinct epigenetic modifications share HDMSs, we further investigated the correlation between the dynamics scores of different epigenetic marks. As shown in Fig. 3, the investigated epigenetic marks demonstrate varied correlation in the three comparison groups. In particular, the epigenetic marks show higher correlation in the hESCs and hESC-derived precursor group. This result indicates that the dynamics of epigenetic modifications are similar during the cell differentiation process, which is conformed to the results of previous overlaps analysis.

\section{Highly dynamic modification sites are related to various genomic features}

Further, we mapped the identified HDMSs to RefSeq genes and collected the genes enriched with HDMSs for each epigenetic mark. Here we explored how the dynamic epigenome participates in early embryonic developmental stages and focused on the hESCs and hESC-derived precursor group. To examine the potential functions of those genes, we performed systematic gene ontology enrichment analysis using DAVID tools (https://david.ncifcrf. gov/) and summarized the key biological processes and pathways for each epigenetic mark. Overall, for the aforementioned six epigenetic modification marks, we found that those HDMSs-enriched genes exhibit enrichment for cell differentiation and development functions (Table 1$)(p$ value $<0.05$ ). For example, GO terms related to development such as 'nervous system development' are enriched in HDMSs of H3K4me1, H3K4me3, H3K9me3,H3K27ac, H3K27me3, GO terms related to differentiation such as 'neuron differentiation' and 'cerebellar granule cell differentiation' are enriched in HDMSs of H3K4me1, H3K9me3, H3K27me3, H3K36me3. This is consistent with previous finding that regulatory elements essential for cellular identity are often epigenetically modified in parental cells $[28,29]$. The results highlight the importance of stage-specific epigenetic modification patterns of transcription factors for defining the developmental potentials.

Also, we noticed that the biological processes of distinct epigenetic marks have overlappings. One possible interpretation for this observation could be that these epigenetic marks may have the same changing trend, collaborating with each other to finish the complex regulatory functions. Taken together, the above results of GO annotation demonstrated the power of our method in identifying the highly dynamic sites of these epigenetic modifications. And, the results strongly suggest that the HDMSs mark critical regulatory regions for cell differentiation and development process. Further characterization

\begin{tabular}{|c|c|c|c|c|c|c|c|c|c|c|c|c|c|c|c|c|c|}
\hline \multicolumn{6}{|l|}{$\mathbf{A}$} & \multicolumn{6}{|l|}{ B } & \multicolumn{6}{|l|}{$\mathrm{C}$} \\
\hline $100 \%$ & $50 \%$ & $43 \%$ & $57 \%$ & $49 \%$ & $40 \%$ H3K4me 1 & $100 \%$ & $25 \%$ & $22 \%$ & $25 \%$ & $20 \%$ & $25 \%$ H3K4me 1 & $100 \%$ & $21 \%$ & $19 \%$ & $22 \%$ & $21 \%$ & $24 \%$ H3K4me1 \\
\hline $48 \%$ & $100 \%$ & $48 \%$ & $57 \%$ & $54 \%$ & $45 \%$ H3к4me3 & $17 \%$ & $100 \%$ & $25 \%$ & $21 \%$ & $21 \%$ & $21 \%$ H3K4me3 & $15 \%$ & $100 \%$ & $18 \%$ & $17 \%$ & $21 \%$ & $19 \%$ н3к4me3 \\
\hline $41 \%$ & $48 \%$ & $100 \%$ & $51 \%$ & $49 \%$ & 39\% нзк9те 3 & $15 \%$ & $24 \%$ & $100 \%$ & $16 \%$ & $25 \%$ & $19 \%$ нзк9тез & $17 \%$ & $22 \%$ & $100 \%$ & $19 \%$ & $23 \%$ & $22 \%$ нзк9mез \\
\hline $48 \%$ & $49 \%$ & $43 \%$ & $100 \%$ & $48 \%$ & $39 \%$ н3к27ас & $23 \%$ & $29 \%$ & $24 \%$ & $100 \%$ & $18 \%$ & $25 \%$ Н3к27ас & $22 \%$ & $23 \%$ & $20 \%$ & $100 \%$ & $21 \%$ & $24 \%$ Н3к27ас \\
\hline $46 \%$ & $52 \%$ & $48 \%$ & $55 \%$ & $100 \%$ & $41 \%$ H3K $27 \mathrm{me} 3$ & $13 \%$ & $20 \%$ & $24 \%$ & $12 \%$ & $100 \%$ & $15 \%$ H3K $27 \mathrm{me} 3$ & $16 \%$ & $22 \%$ & $19 \%$ & $17 \%$ & $100 \%$ & $19 \%$ H3K $27 \mathrm{me} 3$ \\
\hline 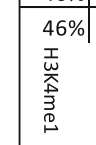 & 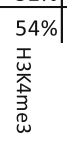 & 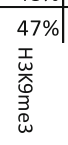 & $\begin{array}{l}56 \% \\
\frac{I}{\omega} \\
\text { N } \\
\stackrel{N}{N}\end{array}$ & 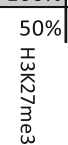 & 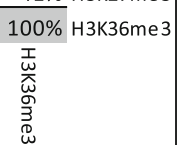 & 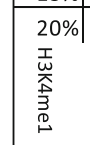 & 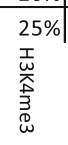 & 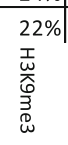 & 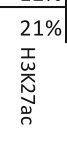 & 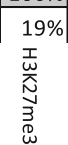 & 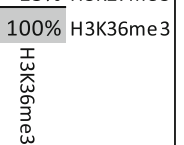 & 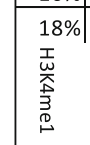 & 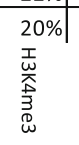 & 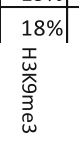 & $\begin{array}{l}19 \% \\
\frac{T}{\omega} \\
\underline{N} \\
\stackrel{N}{N}\end{array}$ & 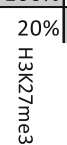 & 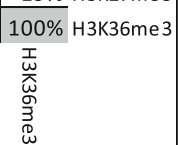 \\
\hline
\end{tabular}

Fig. 2 The overlaps between the top HDMSs of different epigenetic marks. The value in row i column j represents the proportion of HDMSs of epigenetic modification i overlapped by those of epigenetic modification j. a The hESCs and hESC-derived precursor group. b The primary tissues group. c The whole group 


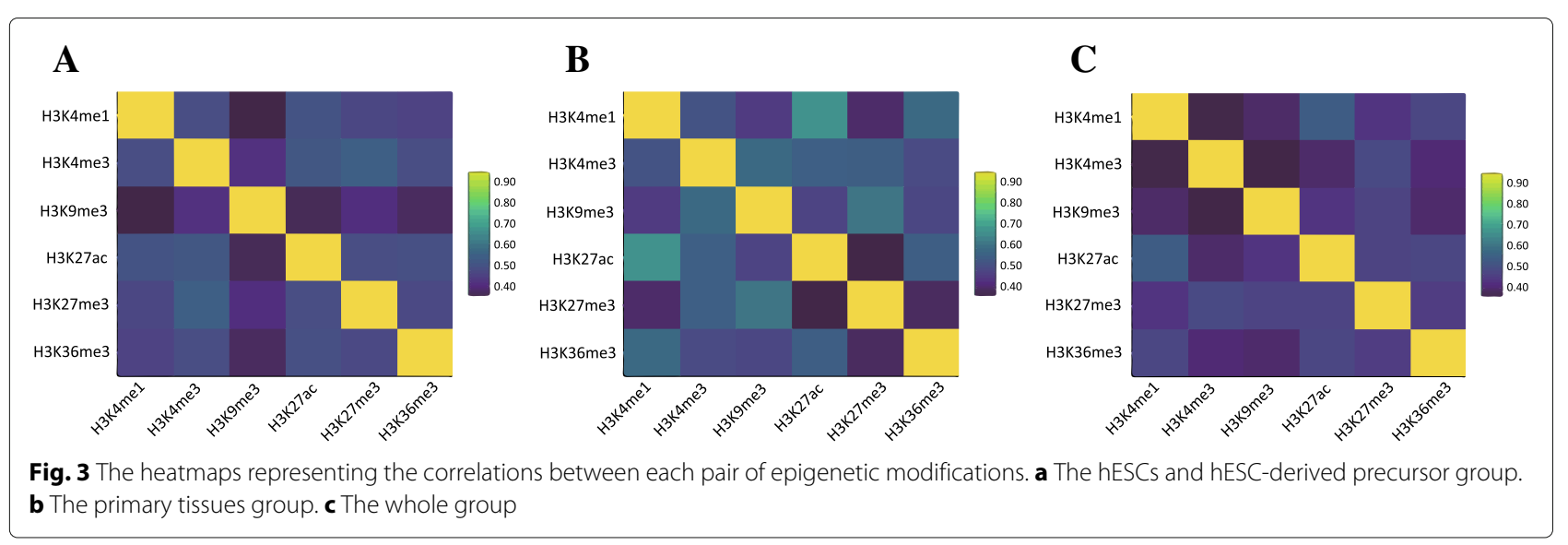

of epigenetic modification patterns and gene expression within HDMSs may provide important insights into the regulatory functions of the specific epigenetic patterns.

\section{Highly dynamic modified sites neighboring genes reveal diverse transcriptional patterns}

To analyze the regulatory roles of these dynamic epigenetic patterns, we further explored the epigenetic modification and gene expression patterns within HDMSs. We computed the correlation coefficients between the dynamics of epigenetic modifications and gene expression levels of the HDMSs-enriched genes. We mapped the HDMSs to Ref-Seq genes and obtained gene expression of the associated genes. As these 20 cell types were divided into three groups, the dynamics score of gene expression was assessed using the same method as epigenetic marks (see Methods). For those HDMSs located in promoters, and coding regions, the Pearson correlation coefficients were respectively computed.

As shown in Fig. 4, we noted that there is highly correlation between the dynamics of gene expression level and epigenetic modification in promoter regions. Relatively, the correlation in coding regions is lower. These results indicate that the variance of epigenetic modification patterns in promoter regions has a higher regulatory role than that in coding regions. The three different groups have a similar trend. In detail, the six epigenetic modification marks exhibit different regulatory effect. For the $\mathrm{hESCs}$ and hESC-derived precursor group, the dynamics of gene expression levels are highly regulated by the modification patterns of H3K4me1 and H3K27me3 in promoter regions. For the primary tissues, the correlations are much higher for H3K9me3 and H3k27ac.

\section{Comparison with QDMR and IOD in identifying HDMSs}

Considering that our method was developed for the differential analysis for multiple cell types, we compared DiffEM with two similar previous methods QDMR and IOD $[22,27]$, which were also designed for multiple conditions.
QDMR is based on Shannon entropy [22], and IOD is defined as the variance divided by the mean value [27]. The performance was measured by the correlation analysis between the epigenetic modification dynamics and gene expression difference.

Firstly, we respectively identified the highly dynamic modification sites using these three methods, and ranked the HDMSs according to the dynamics score. Similarly, we obtained the ranked highly dynamic expression sites. Then, we associated these HDMSs with the highly dynamic expression sites by bitwise matching. To evaluate the performance of these three methods, we define two metrics, MatchedNum and AveDS. MatchedNum is computed as the number of highly dynamic expression sites matching with the top ranked HDMSs, which is similar to recall. AveDS represents the average dynamics score of these matched highly dynamic expression sites. Here, for fair comparison among the three methods, we calculated the entropy as the average dynamics score as QMDR.

We compared the performance on the aforemetioned 6 epigenetic modifications, the results are shown in Fig. 5 and Additional file 1. Figure 5 shows the comparison results for the hESCs and hESC-derived precursor group. Figure S1, Figure S2 (see Additional file 1) showed the results of the other two groups. We first compared the matched numbers of all differential gene expression sites output by these methods. Our method could get a higher MatchedNum of highly dynamic expression sites than those of QDMR and IOD (Fig. 5a). However, this raises the question that to what extent these matched sites are dynamically expressed. As we noted that changes in epigenetic modifications could cause differential expression of related genes, we further compared the average dynamics of gene expression of these matched sites. Lower ave indicate better performance. As the results showed (Fig. 5b), our method has good performance in AveDS. These observations demonstrate that our method always achieves a balance between matched MatchedNum and AveDS, which means our approach could be applied to 
Table 1 Functional enrichment of genes on the whole genome of six histone modifications

\begin{tabular}{|c|c|c|c|c|c|}
\hline Term type & Term name & $P$-value & Term type & Term name & $P$-value \\
\hline \multicolumn{6}{|l|}{ H3K4me1 } \\
\hline $\mathrm{BP}$ & Cell adhesion & 1.42E-06 & CC & Cytoskeleton & 2.90E-03 \\
\hline BP & Axon guidance & 2.86E-05 & CC & Growth cone & 2.15E-02 \\
\hline BP & Nervous system development & $1.91 \mathrm{E}-04$ & KEGG & Arrhythmogenic right ventricular & $5.82 \mathrm{E}-03$ \\
\hline BP & Signal transduction & 1.92E-04 & & Cardiomyopathy (ARVC) & \\
\hline BP & Neuron development & $2.68 \mathrm{E}-02$ & KEGG & Axon guidance & 2.79E-02 \\
\hline BP & Cerebellar granule cell differentiation & 4.35E-02 & KEGG & Hippo signaling pathway & 4.32E-02 \\
\hline \multicolumn{6}{|l|}{ H3K4me3 } \\
\hline BP & Intracellular signal transduction & 7.16E-04 & BP & Adult behavior & $6.93 \mathrm{E}-03$ \\
\hline BP & Signal transduction & $1.22 \mathrm{E}-03$ & MF & Extracellular-glutamate-gated ion channel & 2.77E-03 \\
\hline BP & Nervous system development & $2.63 \mathrm{E}-03$ & & Activity & \\
\hline BP & Chemical synaptic transmission & 2.88E-02 & KEGG & Neuroactive ligand-receptor interaction & $6.24 \mathrm{E}-03$ \\
\hline \multicolumn{6}{|l|}{ H3K9me3 } \\
\hline BP & Heterophilic cell-cell adhesion & 1.87E-07 & $\mathrm{BP}$ & Regulation of RNA splicing & $9.41 \mathrm{E}-03$ \\
\hline BP & Cell adhesion & 1.06E-04 & $\mathrm{BP}$ & Regulation of alternative mRNA splicing & $1.58 \mathrm{E}-02$ \\
\hline BP & Nervous system development & $6.27 \mathrm{E}-04$ & $\mathrm{BP}$ & Chemical synaptic transmission & $3.35 \mathrm{E}-02$ \\
\hline \multirow[t]{2}{*}{ BP } & Regulation of neuron projection & 4.09E-03 & BP & Cerebellar granule cell differentiation & $3.98 \mathrm{E}-02$ \\
\hline & Development & & MF & Calcium ion binding & 1.25E-05 \\
\hline BP & Signal transduction & $5.41 \mathrm{E}-03$ & KEGG & Cell adhesion molecules (CAMs) & 2.10E-02 \\
\hline \multicolumn{6}{|l|}{ H3K27ac } \\
\hline BP & Signal transduction & 7.03E-05 & $\mathrm{BP}$ & Regulation of RNA splicing & $2.08 \mathrm{E}-02$ \\
\hline BP & Nervous system development & 7.47E-05 & BP & Cytoskeleton organization & 3.57E-02 \\
\hline BP & Neuron cell-cell adhesion & 2.31E-04 & MF & Actin binding & 1.20E-04 \\
\hline BP & Neuron development & $5.28 \mathrm{E}-03$ & CC & Growth cone & 1.83E-04 \\
\hline BP & Glutamate receptor signaling pathway & $5.76 \mathrm{E}-03$ & MF & Protein kinase activity & $1.15 \mathrm{E}-02$ \\
\hline BP & Brain development & 1.57E-02 & KEGG & Neuroactive ligand-receptor interaction & $1.06 \mathrm{E}-02$ \\
\hline \multicolumn{6}{|l|}{ H3K27me3 } \\
\hline BP & Social behavior & $9.15 E-05$ & BP & Cerebellar granule cell differentiation & $3.89 \mathrm{E}-02$ \\
\hline BP & Signal transduction & 5.10E-04 & MF & Calcium ion binding & 7.03E-05 \\
\hline BP & Nervous system development & 2.97E-03 & MF & Cell adhesion molecule binding & 1.65E-04 \\
\hline BP & Regulation of RNA splicing & 8.99E-03 & CC & Growth cone & $1.55 \mathrm{E}-02$ \\
\hline \multicolumn{6}{|l|}{ H3K36me3 } \\
\hline BP & Heterophilic cell-cell adhesion & 4.04E-07 & CC & Neuron projection & 4.32E-03 \\
\hline BP & Signal transduction & 2.27E-03 & MF & Actin binding & 7.37E-03 \\
\hline BP & Cell adhesion & $1.25 \mathrm{E}-02$ & KEGG & Neuroactive ligand-receptor interaction & 4.97E-02 \\
\hline BP & Neuron differentiation & 3.37E-02 & & & \\
\hline
\end{tabular}

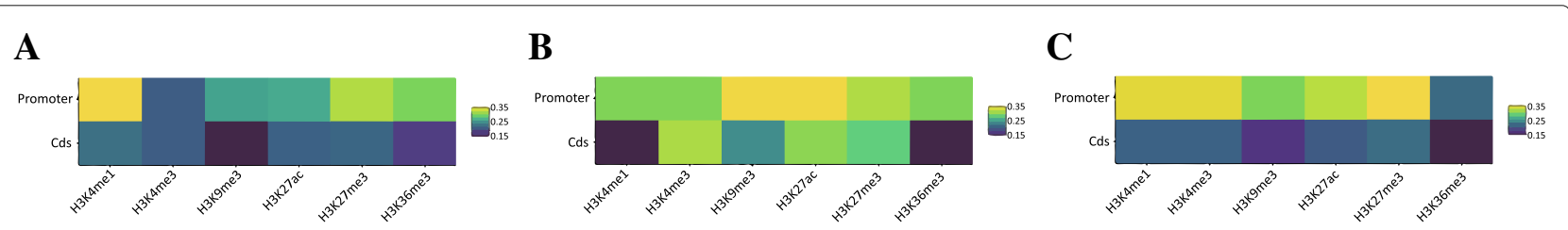

Fig. 4 The correlations between the dynamics of epigenetic modification and that of expression level in HDMSs. a The hESCs and hESC-derived precursor group. $\mathbf{b}$ The primary tissues group. $\mathbf{c}$ The whole group 


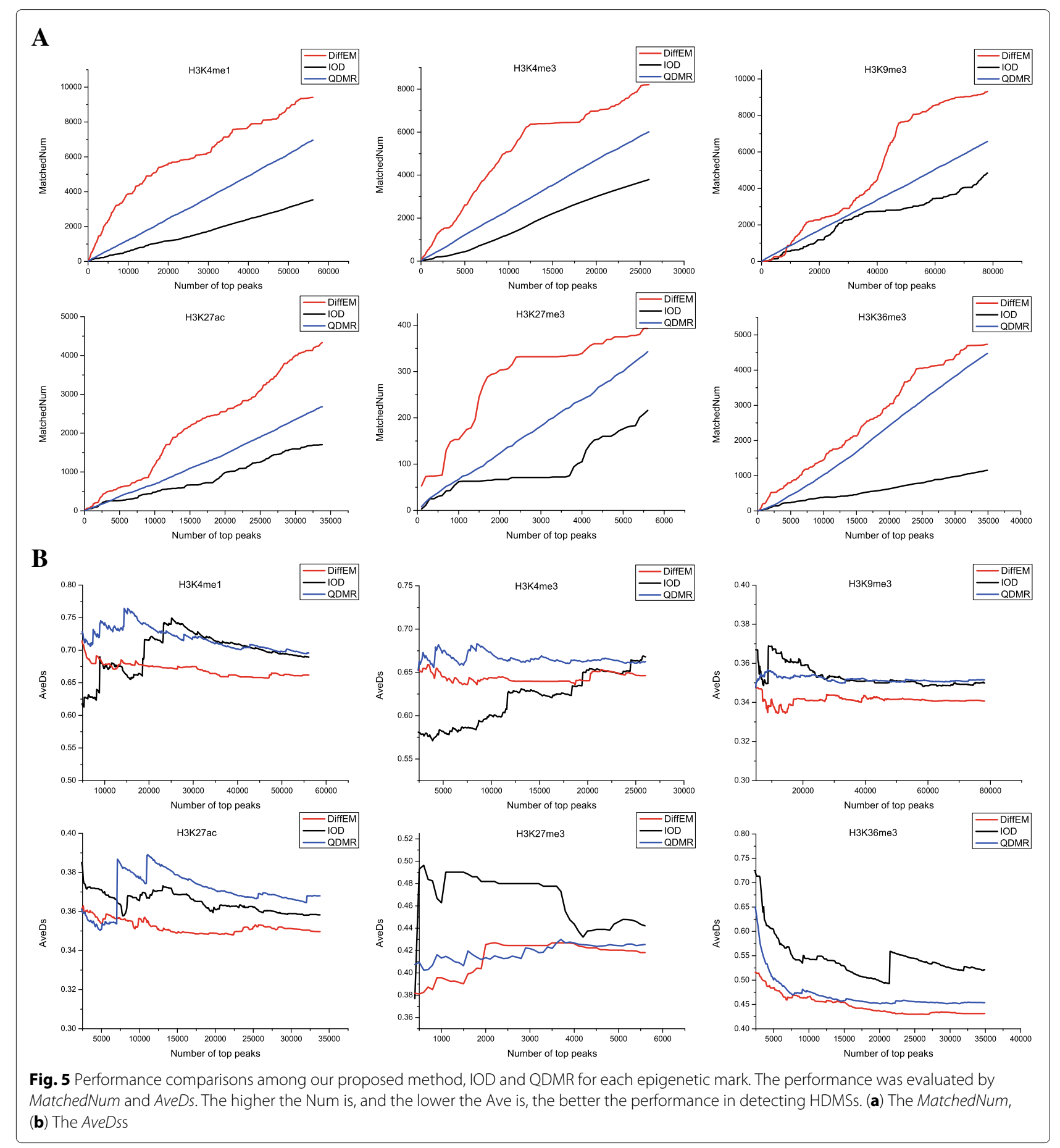

find meaningful HDMSs as many as possible. In addition, the overall analysis for MatchedNum and AveDS shows that IOD may be applicable to detecting the highest HDMSs, because of the commonly small Num but better AveDS of related differential gene expression sites. In summary, our method outperforms the two existing methods in identifying the HDMSs across different developmental stages and tissues in the whole genome.

\section{Discussion}

In this paper, we proposed a new computational method, DiffEM, based on hamming distance to identify the highly dynamic modification sites that undergo chromatin changes during human cell differentiation process. Different from previous methods that mostly focused on differential analysis between two cell types, our method is designed for differential analysis of genome- 
wide epigenetic modification across multiple cell types. DiffEM can be broadly applied in a range of studies involving various epigenetic marks in different conditions. We applied this approach to investigating 6 epigenetic marks of 20 human cell types, including hESCs, 4 hESC-derived Lineages and 15 human primary tissues. We identified highly dynamic modification sites where different cell types exhibit distinctive epigenetic modification patterns, and found that these highly dynamic modification sites are enriched in the genes are related to cellular development and differentiation. The results also demonstrate the strong association among the dynamics of different epigenetic marks, consistent with previous finding that different epigenetic modifications collaborate with each other to consummate complex regulatory functions. Further, we evaluated the effectiveness of our method, by correlating the dynamics scores of epigenetic modification with the variance of gene expression. We compared DiffEM with two existing methods, QDMR and IOD. The comparison results indicate the power of our method in quantifying the epigenetic dynamics and identifying highly dynamic regions.

\section{Additional file}

Additional file 1: Figure S1. Performance comparisons for primary tissues among our method DiffEM, IOD and QDMR for each epigenetic mark. (A) The MatchedNum, (B) The AveDS. Figure S2. Performance comparisons for the whole group among our method DiffEM, IOD and QDMR for each epigenetic mark. (A) The MatchedNum, (B) The AveDS. (PDF $405 \mathrm{~kb}$ )

\section{Abbreviations}

DAVID: The database for annotation, visualization and integration discovery; GO: Gene ontology; HDMSs: Highly dynamic modification sites; hESCs: Human embryonic stem cells; iHMS: A database integrating human histone modification data across developmental stages and tissues; ME: Mesendoderm; MSCs: Mesenchymal stem cells; NPCs: Neural progenitor cells; TBL: Trophoblast-like cells

\section{Acknowledgements}

Authors are grateful to NIH Roadmap Epigenome Project and iHMS website for providing the epigenomic data to carry out this work.

\section{Funding}

This work and the publication costs were supported in part by the Fundamental Research Funds for the Central Universities (2232016A3-05), the National Natural Science Foundation of China (61772128, 61772367), National Key Research and Development Program of China (2016YFC0901704) and Shanghai Natural Science Foundation (17ZR1400200,18ZR1414400).

\section{Availability of data and material}

Supplementary data are available at Github: https://github.com/ xiazhang0513/DiffEM.

\section{About this supplement}

This article has been published as part of BMC Genomics Volume 20 supplement 2, 2019: Selected articles from the 17th Asia Pacific Bioinformatics Conference (APBC 2019): genomics. The full contents of the supplement are available online at https://bmcgenomics.biomedcentral.com/articles/supplements/volume20-supplement-2.

\section{Authors' contributions}

YLG and $X Z$ are responsible for the main idea, as well as the completion of the manuscript and experiment. GBZ, SGZ and JHG have coordinated data preprocessing and supervised the effort. All authors have read and approved the final manuscript.

\section{Ethics approval and consent to participate}

Not applicable.

Consent for publication

Not applicable.

\section{Competing interests}

The authors declare that they have no competing interests.

\section{Publisher's Note}

Springer Nature remains neutral with regard to jurisdictional claims in published maps and institutional affiliations.

\section{Author details}

${ }^{1}$ School of Computer Engineering and Science, Shanghai University, Shanghai, China. ${ }^{2}$ School of Computer Science and Technology, Donghua University, Shanghai, China. ${ }^{3}$ Department of Computer Science and Technology,Tongji University, Shanghai, China. ${ }^{4}$ Shanghai Key Lab of Intelligent Information Processing, and School of Computer Science, Fudan University, Shanghai, China.

Published: 10 April 2019

\section{References}

1. Atlasi Y, Stunnenberg HG. The interplay of epigenetic marks during stem cell differentiation and development. Nat Rev Genet. 2017;18(6990): 643-58.

2. Kouzarides T. Chromatin modifications and their function. Cell. 2007;128: 693-705.

3. Rivera CM, Ren B. Mapping human epigenomes. Cell. 2013;155(1):39-55.

4. Dixon JR, Jung I, Selvaraj S, Shen Y, Antosiewicz-Bourget JE, Lee AY, et al. Chromatin architecture reorganization during stem cell differentiation. Nature. 2015;518(7539):331-6.

5. Mikkelsen TS, Ku M, Jaffe DB, Issac B, Lieberman E, Giannoukos G, Alvarez P, Brockman W, Kim TK, Koche RP. Genome-wide maps of chromatin state in pluripotent and lineage-committed cells. Nature. 2007:448(7153):553.

6. Tsankov AM, et al. Transcription factor binding dynamics during human es cell differentiation. Nature. 2015;518(7539):344-9.

7. Ernst J, Kheradpour P, Mikkelsen TS, Shoresh N, Ward LD, Epstein CB, Zhang X, Wang L, Issner R, Coyne M, et al. Mapping and analysis of chromatin state dynamics in nine human cell types. Nature. 2011;473(7345):43-9.

8. Roadmap EC, Kundaje A, Meuleman W, Ernst J, et al. Integrative analysis of 111 reference human epigenomes. Nature. 2015;518(7539):317-30.

9. Gifford CA, Ziller MJ, Gu H, Trapnell C, Donaghey J, Tsankov A, Shalek AK, Kelley DR, Shishkin AA, Issner R, et al. Transcriptional and epigenetic dynamics during specification of human embryonic stem cells. Cell. 2013;153(5):1149-63.

10. Xie W, Schultz MD, Lister R, et al. Epigenomic analysis of multilineage differentiation of human embryonic stem cells. Cell. 2013;153(5):1134-48.

11. Thomson JA, Itskovitz-Eldor J, Shapiro SS, Waknitz MA, Swiergiel JJ, Marshall VS, Jones JM. Embryonic stem cell lines derived from human blastocysts. Science. 1998;282(5391):1145.

12. Vodyanik MA, Yu J, Zhang X, Tian S, Stewart R, Thomson JA, Slukvin II. A mesoderm-derived precursor for mesenchymal stem and endothelial cells. Cell Stem Cell. 2010;7(6):718.

13. Yu P, Xiao S, Xin X, Song C-X, Huang W, McDee D, Tanaka T, Wang T, He C, Zhong S. Spatiotemporal clustering of the epigenome reveals rules of dynamic gene regulation. Genome Res. 2013;23(2):352-64.

14. Xu H, Wei CL, Lin F, Sung WK. An hmm approach to genome-wide identification of differential histone modification sites from chip-seq data. Bioinformatics. 2008;24(20):2344-9.

15. Shen L, Shao NY, Liu X, Maze I, Feng J, Nestler EJ. diffreps: Detecting differential chromatin modification sites from chip-seq data with biological replicates. PloS ONE. 2013;8(6):65598. 
16. Ji H, Li X, Wang QF, Ning Y. Differential principal component analysis of chip-seq. Proc Natl Acad Sci U S A. 2013;110(17):6789.

17. Heinig M, Colom-Tatch M, Taudt A, Rintisch C, Schafer S, Pravenec M, Hubner N, Vingron M, Johannes F. histonehmm: Differential analysis of histone modifications with broad genomic footprints. BMC Bioinformatics. 2015;16(1):1-15.

18. Lun ATL, Smyth GK. csaw: a bioconductor package for differential binding analysis of chip-seq data using sliding windows: Nucleic Acids Res. 2016;44(5):45.

19. Ashoor H, Louis-Brennetot C, Janoueix-Lerosey I, Bajic VB, Boeva V. Hmcan-diff: a method to detect changes in histone modifications in cells with different genetic characteristics. Nucleic Acids Res. 2017;45(8):58.

20. Chen C, Zhang S, Zhang XS. Discovery of cell-type specific regulatory elements in the human genome using differential chromatin modification analysis. Nucleic Acids Res. 2013;41(20):9230.

21. Yang X, Shao X, Gao L, Zhang S. Systematic dna methylation analysis of multiple cell lines reveals common and specific patterns within and across tissues of origin. Hum Mol Genet. 2015;24(15):4374.

22. Zhang Y, Liu H, Lv J, Xiao X, Zhu J, Liu X, Su J, Li X, Wu Q, Wang F. Qdmr: a quantitative method for identification of differentially methylated regions by entropy. Nucleic Acids Res. 2011;39(9):58.

23. Bhasin JM, Bo H, Ting AH. Methylaction: detecting differentially methylated regions that distinguish biological subtypes. Nucleic Acids Res. 2016;44(1):106-16.

24. Gan Y, Han T, Guan J, Zhou S. ihms: a database integrating human histone modification data across developmental stages and tissues. BMC Bioinformatics. 2017;18(1):103.

25. Gan Y, Tao H, Zou G, Yan C, Guan J. Dynamic epigenetic mode analysis using spatial temporal clustering. BMC bioinformatics. 2016;17(17):537.

26. Zhang Y, An L, Yue F, Hardison RC. Jointly characterizing epigenetic dynamics across multiple human cell types. Nucleic Acids Res. 2016:44(14):6721-31.

27. Pinello L, Xu J, Orkin SH, Yuan GC. Analysis of chromatin-state plasticity identifies cell-type-specific regulators of h3k27me3 patterns. Proc Natl Acad Sci U S A. 2014;111(3):344.

28. Wang H, Liu C, Deng L. Enhanced prediction of hot spots at protein-protein interfaces using extreme gradient boosting. Sci Rep. 2018;8(1):14285.

29. Pan Y, Liu D, Deng L. Accurate prediction of functional effects for variants by combining gradient tree boosting with optimal neighborhood properties. PloS ONE. 2017;12(6):0179314.

Ready to submit your research? Choose BMC and benefit from:

- fast, convenient online submission

- thorough peer review by experienced researchers in your field

- rapid publication on acceptance

- support for research data, including large and complex data types

- gold Open Access which fosters wider collaboration and increased citations

- maximum visibility for your research: over 100M website views per year

At $\mathrm{BMC}$, research is always in progress.

Learn more biomedcentral.com/submissions 